\title{
Neumonía grave del adulto adquirida en la comunidad manejada en sala de cuidados generales en un hospital público
}

\author{
EDUARDO CEA V.*, GONZALO ATLAGICH G.*, MARCO NÚÑEZ C.*, \\ KATHERINE CHÁVEZ R.*, MAYRA CALDERÓN Q.* y VICTORIA NOVIK A.**
}

\begin{abstract}
Severe community-acquired pneumonia in adult patients managed in a general ward at a public hospital
\end{abstract}

Introduction: Severe community-acquired pneumonia (severe CAP) is characterized by intensive care unit (ICU) requirement, given its higher mortality and complications rate. Different prediction scales have been designed to assess severity. Objective: Identify adult patients with severe CAP managed in the general ward of a public hospital. Patients and Methods: Retrospective descriptive study, including all adult patients discharged from hospital with CAP diagnosis, from January $1^{\text {st }}$ to December $31^{\text {st }}$,2010, with a total of 131 medical records reviewed. The American Thoracic Society criteria (ATS) of severe CAP and CURB-65 scores from 89 medical records were calculated. Results: 16 patients (18\%) had CURB-65 score $\geq 3,8$ patients (9\%) had $\geq 3$ minor ATS criteria, and 2 of those patients had a major criterion. Conclusion: A significant number of patients with severe CAP were managed on the general ward of a public hospital.

Key words: Severe community acquired pneumonia, CURB-65, ATS criteria, general ward, prognosis, outcome.

\section{Resumen}

Introducción: La neumonía grave del adulto adquirida en la comunidad (NAC grave) se caracteriza por requerir manejo en la unidad de cuidados intensivos (UCI), dado su mayor mortalidad y riesgo de complicaciones, por lo cual se han diseñado distintas escalas de predicción de gravedad. Objetivo: Identificar a pacientes adultos con NAC grave manejados en la sala de cuidados generales. Pacientes y Métodos: Estudio clínico descriptivo retrospectivo, se revisaron las fichas clínicas de 131 pacientes adultos egresados del servicio de medicina del hospital de Quilpué con el diagnóstico de $N A C$ entre el 1 de enero y 31 de diciembre del 2010. Se examinaron los criterios de NAC grave de la Sociedad de Tórax Americana (ATS) y se midió el CURB-65 de 89 pacientes que consignaban esta información. Resultados: En 16 pacientes (18\%) se obtuvo una puntuación del CURB-65 $\geq 3$ y en 8 pacientes (9\%) se identificaron $\geq 3$ criterios menores de la ATS y en 2 de ellos un criterio mayor. Conclusión: Se identificaron pacientes con NAC grave manejados en la sala de cuidados generales de un hospital público.

Palabras clave: Neumonía grave adquirida en la comunidad, CURB-65, criterios ATS, sala de cuidados generales, pronóstico, resultado.

\section{Introducción}

Se entiende por neumonía grave del adulto adquirida en la comunidad (NAC grave) a aquella que requiere para su manejo y/o vigilancia, una unidad de cuidados intensivos (UCI), para que permita, si es necesario, apoyo con ventilador mecánico y/o soporte hemodinámico ${ }^{1}$. La tasa de hospitalización anual en Estados Unidos por neumonía adquirida en la comunidad (NAC) fluctúa entre 0,4 y 4 casos por 1.000 personas $^{2}$. De las cuales el 10 a 30\% requerirán ser manejados en

\footnotetext{
* Facultad de Medicina, Universidad Andrés Bello, Sede Viña del Mar, Chile.

** Departamento de Medicina Interna, Facultad de Medicina, Universidad Andrés Bello, Sede Viña del Mar.
} 
una $\mathrm{UCI}^{3-7}$. La mortalidad de los pacientes con NAC grave varía entre $21 \%$ y $54 \% 0^{5,6,8}$, lo que hace imprescindible un diagnóstico correcto de la gravedad para el manejo adecuado.

Para objetivar el diagnóstico de NAC grave se han desarrollado distintos sistemas de puntaje y modelos predictores para la identificación y diagnóstico precoz de esta entidad. Entre ellos, destacan los criterios de la American Thoracic Society (ATS) ${ }^{6}$ y los criterios de la British Thoracic Society (BTS) ${ }^{7}$.

Consideramos que la NAC grave es subdiagnosticada en nuestro medio, no existiendo uniformidad en el diagnóstico a nivel nacional, impidiendo un manejo adecuado acorde con las normas nacionales e internacionales ${ }^{5-7,9,10}$. Por lo anterior, en el presente trabajo realizamos un análisis retrospectivo y descriptivo para identificar y calificar la severidad de los pacientes adultos hospitalizados por neumonía adquirida en la comunidad (NAC), ingresados durante el año 2010 en el hospital de Quilpué.

\section{Pacientes y Métodos}

Se realizó un estudio clínico descriptivo retrospectivo de los pacientes adultos egresados del Hospital de Quilpué con diagnóstico de neumonía comunitaria entre el $1^{\circ}$ de enero y el 31 de diciembre del 2010. El Hospital de Quilpué es un hospital de mediana complejidad sin disponibilidad de UCI. Los pacientes de mayor gravedad son manejados en la unidad de paciente crítico (UPC), y eventualmente derivados al centro de referencia, Hospital Gustavo Fricke de Viña del Mar, en caso de necesitar UCI. Se encuentra situado en la comuna de Quilpué, con una población asignada de 128.578 habitantes ${ }^{11}$.

De un total de 131 pacientes adultos $(>18$ años) egresados con diagnóstico de NAC, se accedió a la totalidad de las fichas clínicas. Fueron excluidos de este estudio los pacientes con condiciones previas que pudieran interferir con los parámetros a analizar: paciente sin datos de ingreso, con inmunosupresión manifiesta, insuficiencia renal crónica (IRC) consignada en la ficha clínica independiente de la etapa, cáncer de cualquier etiología, foco infeccioso concomitante y adecuación del esfuerzo terapéutico descrito en la ficha clínica. El número total de fichas clínicas válidas para el análisis fue 89 (67,9\%). Las características de la población excluida se describen en la Tabla 1.

De las 89 fichas clínicas, se recopilaron los siguientes datos consignados en la admisión al hospital: edad, género, frecuencia respiratoria al ingreso, presencia de infiltrado multilobar en la radiografía de tórax, presencia de confusión y/o desorientación, nitrógeno ureico sérico elevado (mayor de $20 \mathrm{mg} / \mathrm{dL}$ ), leucocitopenia (menor de $4.000 / \mathrm{mm}^{3}$ ), trombocitopenia (menor de $100.000 /$ $\mathrm{mm}^{3}$ ), presencia de hipotermia (temperatura menor de $36{ }^{\circ} \mathrm{C}$ ), hipotensión arterial (PAS menor de $90 \mathrm{mmHg}$ y/o PAD menor o igual a $60 \mathrm{mmHg}$ ), relación presión arterial de oxígeno y fracción inspirada de oxígeno $\left(\mathrm{PaO}_{2} / \mathrm{FiO}_{2}\right)$, shock séptico con uso de droga vasoactiva, uso de ventilación mecánica, ingreso a UPC, días de hospitalización y pacientes fallecidos en el hospital. Estos datos se sustentan en los parámetros expuestos tanto por la $\mathrm{ATS}^{5}$ y $\mathrm{BTS}^{6}$ para la evaluación de la gravedad de la neumonía comunitaria, además de los datos clínicos relevantes para efectuar una caracterización epidemiológica.

Se calculó el puntaje ATS y CURB-65, donde se consideró, para ambos índices pronósticos, un puntaje $\geq 3$ o algún criterio mayor de la ATS, como criterio diagnóstico de NAC grave $e^{5,6,7,9}$.

\section{Análisis estadístico}

Los datos extraídos fueron tabulados en MS Excel 2010 y se analizaron con el programa SPSS Versión 17.0. Las pruebas estadísticas utilizadas fueron la prueba de chi-cuadrado y el coeficiente de correlación de Pearson. Se consideró significativo un valor de $\mathrm{p}<0,05$.

\section{Resultados}

De los 89 pacientes, 49 fueron mujeres $(55,1 \%)$ y 40 hombres $(44,9 \%)$. La edad media fue de 70,9 años con una distribución etaria entre los 21 y los 106 años. La media de días de hos-

\section{Tabla 1. Características clínicas de los pacientes hospitalizados por neumonía comunitaria excluidos del estudio}

\begin{tabular}{|lrr|}
\hline & $\mathbf{n}$ & $(\mathbf{( \% )}$ \\
Total & 42 & $(100)$ \\
Masculino/Femenino & \multicolumn{2}{c|}{$24 / 18$} \\
Sin información & 14 & $(33,3)$ \\
Insuficiencia renal crónica & 9 & $(21,4)$ \\
Cáncer & 3 & $(7,1)$ \\
Foco infeccioso concomitante & 3 & $(7,1)$ \\
Inmunodepresión & 7 & $(16,6)$ \\
Manejo proporcional & 6 & $(14,2)$ \\
\hline
\end{tabular}




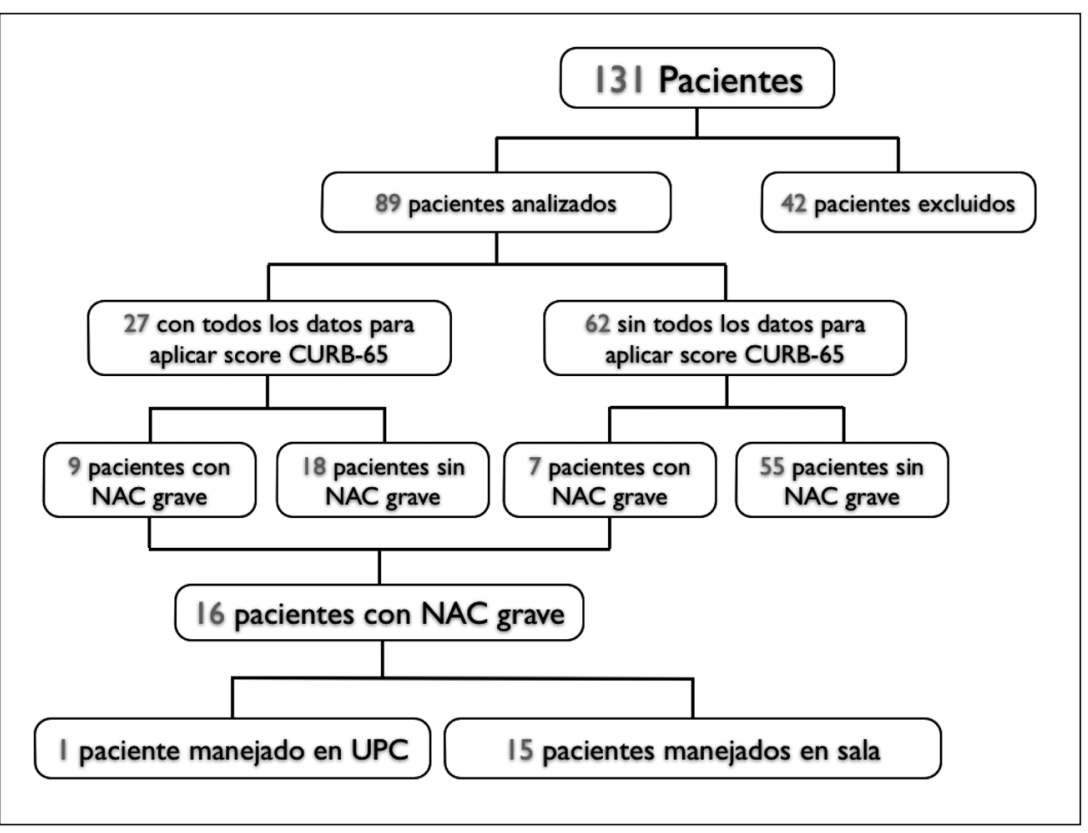

Figura 1. Manejo de pacientes adultos hospitalizados por neumonía comunitaria según el índice pronóstico de la Sociedad Británica de Tórax (CURB-65). pitalización fue de 7,9 días con un mínimo de 2 días y máximo de 23 días. No hubo correlación significativa entre la estadía en el hospital (díascama) y la gravedad de la neumonía $(\mathrm{p}>0,05)$.

De las fichas clínicas analizadas, en sólo 27 pacientes $(30,3 \%)$ se pudo obtener la totalidad de los datos para calcular el puntaje del CURB65 (Figura 1). Ninguna ficha clínica contaba con todos los datos para estimar la gravedad de la neumonía según los criterios de la ATS. Las características clínico-epidemiológicas de la población con NAC grave y NAC no grave manejada en la sala de cuidados generales se describen en la Tabla 2.

Seis pacientes $(6,7 \%)$ fallecieron en el hospital, todos tenían criterios de NAC grave. De ellos, dos pacientes tenían un puntaje de CURB65 igual a 3 , tres pacientes un valor igual a 4 y un paciente el puntaje máximo de 5 puntos. De los pacientes fallecidos, dos pacientes tenían dos criterios menores de la ATS, dos pacientes tenían tres criterios, un paciente tenía cuatro criterios y un paciente tenía cinco criterios menores de la ATS. De los dos pacientes que presentaron un criterio mayor de la ATS, uno de ellos falleció.

Los criterios de neumonía comunitaria grave de la ATS y BTS (CURB-65) se asociaron significativamente a mayor riesgo de muerte en el hospital $(\mathrm{p}<0,001)$.

\section{Discusión}

La incidencia de neumonía grave del adulto adquirida en la comunidad pesquisada en este estudio, fue comparable con la expuesta en la literatura nacional y extranjera $(17,9 \%$ de los pacientes hospitalizados) ${ }^{5-7}$.

Las principales limitaciones del presente estudio fueron el diseño descriptivo retrospectivo, el pequeño tamaño muestral y la ausencia de información completa de algunas fichas clínicas, con pérdida de información relevante para el posterior desarrollo y análisis de los resultados.

De las fichas analizadas, se encontró en un tercio de los casos la totalidad de la información requerida para calcular el puntaje del CURB-65. Esto refleja la baja frecuencia de registros clínicos completos imposibilitando el uso de este índice pronóstico validado en el ámbito nacional e internacional para evaluar la gravedad de la NAC, lo que es concordante con lo publicado en la literatura internacional ${ }^{6,7,9}$. En el $69,7 \%$ de las fichas clínicas restantes, se pudo haber subestimado la presencia de NAC grave, con valores estimados del CURB-65 inferiores al valor real (Tabla 3).

Pese a lo anterior, se pesquisaron 16 pacientes con NAC grave según el índice pronóstico de la BTS (CURB-65) que no fueron manejados en unidad de paciente crítico según las recomendaciones de las guías clínicas internacionales y nacionales ${ }^{5-7,9}$. Cabe destacar que se ha descrito en distintas publicaciones que los médicos no utilizan criterios uniformes para valorar el riesgo de morbilidad y mortalidad de los enfermos con neumonía comunitaria, lo cual se asocia a gran variabilidad en la tasas de admisión a sala de cuidados generales y UCI en diferentes áreas 
Tabla 2. Características clínico epidemiológicas de pacientes hospitalizados por neumonía comunitaria en la sala de cuidados generales del Hospital de Quilpué

\begin{tabular}{|c|c|c|c|c|}
\hline \multirow[b]{2}{*}{$\mathrm{N}^{0}$ total $(\%)$} & \multicolumn{2}{|c|}{ NAC no grave } & \multicolumn{2}{|c|}{ NAC grave } \\
\hline & 73 & $(100)$ & 16 & $(100)$ \\
\hline Comorbilidad $\mathrm{N}^{\circ}(\%)$ & 57 & $(78,1)$ & 11 & $(68,8)$ \\
\hline Hipertensión arterial & 39 & $(53,4)$ & 12 & $(75,0)$ \\
\hline Diabetes mellitus tipo 2 & 17 & $(23,3)$ & 3 & $(18,8)$ \\
\hline Accidente cerebrovascular & 2 & $(2,7)$ & 5 & $(31,3)$ \\
\hline Asma & 6 & $(8,2)$ & 0 & $(0,0)$ \\
\hline Tuberculosis & 3 & $(4,1)$ & 1 & $(6,3)$ \\
\hline Cardiopatía & 3 & $(4,1)$ & 1 & $(6,3)$ \\
\hline Enfermedad Alzheimer & 0 & $(0,0)$ & 3 & $(18,8)$ \\
\hline Hipotiroidismo & 1 & $(1,4)$ & 0 & $(0,0)$ \\
\hline Daño hepático crónico & 2 & $(2,7)$ & 0 & $(0,0)$ \\
\hline EPOC & 9 & $(12,3)$ & 1 & $(6,3)$ \\
\hline $\mathrm{FR} \geq 30 \mathrm{resp} / \mathrm{min}$ & 11 & $(15,1)$ & 12 & $(75,0)$ \\
\hline NAC multilobar & 5 & $(6,8)$ & 2 & $(12,5)$ \\
\hline Confusión mental & 6 & $(8,2)$ & 12 & $(75,0)$ \\
\hline $\mathrm{BUN} \geq 20 \mathrm{mg} / \mathrm{dL}$ & 5 & $(6,8)$ & 8 & $(50,0)$ \\
\hline Leucopenia & 3 & $(4,1)$ & 1 & $(6,3)$ \\
\hline Trombocitopenia & 2 & $(2,7)$ & 0 & $(0,0)$ \\
\hline Hipotensión arterial & 7 & $(9,6)$ & 7 & $(43,8)$ \\
\hline Ausencia de fiebre & 0 & $(0,0)$ & 0 & $(0,0)$ \\
\hline Shock séptico & 0 & $(0,0)$ & 1 & $(6,3)$ \\
\hline Unidad paciente crítico & 2 & $(2,7)$ & 2 & $(12,5)$ \\
\hline Fallece en el hospital & 0 & $(0,0)$ & 6 & $(37,5)$ \\
\hline$\geq 65$ años & 45 & $(61,6)$ & 16 & (100) \\
\hline$<65$ años & 28 & $(38,4)$ & 0 & $(0,0)$ \\
\hline Estadía en el hospital & 8,2 & & 6,4 & \\
\hline
\end{tabular}

*EPOC: Enfermedad pulmonar obstructiva crónica, FR: frecuencia respiratoria, NAC: Neumonía comunitaria, BUN: Nitrógeno ureico sérico, leucopenia: $<4.000 \mathrm{cel} / \mathrm{mm}^{3}$.

Tabla 3. Cálculo de CURB-65 y criterios menores de gravedad de la ATS en la admisión al hospital

\begin{tabular}{|ccccc|}
\hline & CURB-65 & $\mathbf{( \% )}$ & ATS & $(\mathbf{\%})$ \\
0 & 21 & $(23,6)$ & 43 & $(48,3)$ \\
1 & 31 & $(34,8)$ & 22 & $(24,7)$ \\
2 & 21 & $(23,6)$ & 16 & $(18,0)$ \\
3 & 10 & $(11,2)$ & 5 & $(5,6)$ \\
4 & 5 & $(5,6)$ & 2 & $(2,2)$ \\
5 & 1 & $(1,1)$ & 1 & $(1,1)$ \\
\hline
\end{tabular}

geográficas $^{5,6,12,13}$. Esta conducta tiene repercusión directa sobre la mortalidad de los pacientes $6,7,9,10$.

Respecto a los criterios de la ATS, ocho pacientes tenían criterios de NAC grave, uno de los cuales presentaba shock séptico con necesidad de uso de droga vasopresora, no fueron manejados acorde a su gravedad ${ }^{5,6}$. Cabe señalar que al igual que con el índice pronóstico de la BTS (CURB-65), por falta de información en las fichas clínicas, un número indeterminado de pacientes pudo haber tenido una NAC grave no diagnosticada.

La totalidad de los pacientes fallecidos en el hospital, se presentó en el grupo de pacientes catalogados como NAC grave, destacando la asociación significativa entre los pacientes fallecidos y el diagnóstico de NAC grave. Esto hace imprescindible la utilización de índices pronósticos validados para la valoración de la gravedad de los enfermos, con el objetivo de derivar oportunamente a unidades especializadas (Unidad de intermedio o UCI) a los pacientes con NAC grave que deben ser manejados en forma adecuada.

Este estudio refleja la importancia de realizar nuevas investigaciones de mayor precisión para determinar la real situación del manejo de pacientes con NAC grave en nuestro medio y su influencia en la mortalidad.

\section{Agradecimientos}

Los autores agradecen a la Dirección del Hospital de Quilpué el apoyo en la ejecución del estudio clínico.

\section{Bibliografía}

1.- LEEPER K V Jr, TORRES A. Communityacquired pneumonia in the intensive care unit. Clin Chest Med 1995; 16: 155-71.

2.- HARRISON B, FARR B, PUGH S, SELKON J B. Community-acquired pneumonia in adults in British hospitals in 1982-1983: a survey of etiology, mortality, prognostic factors and outcome. Q J Med 1987; 62: 195-220.

3.- NIEDERMAN M S. How do we optimize 
outcomes for patients with severe community-acquired pneumonia? Intensive Care Med 2002; 28:1003-5.

4.- ANGUS D C, MARRIE T J, OBROSKY D S, CLERMONT G, DREMSIZOV T T, COLEY C, et al. Severe community-acquired pneumonia: use of intensive care services and evaluation of American and British Thoracic Society Diagnostic criteria. Am J Respir Crit Care Med 2002; 166: 717-23.

5.- MANDELL L A, WUNDERINK R G, ANZUETO A, BARTLETT J G, CAMPBELL G D, DEAN N C, et al. IDSA/ATS Consensus Guidelines on the Management of Community-Acquired Pneumonia. Clin Infect Dis 2007; 44: S27-72.

6.- $\quad$ LIM W S, BAUDOUIN S V, GEORGE R C, HILL A T, JAMIESON C, LE JEUNE I, et al. British Thoracic Society guidelines for the management of community acquired pneumonia in adults: update 2009. Thorax 2009; 64 Suppl 3: iii1-55.

7.- ARANCIBIA F, DÍAZ O. Neumonía grave del adulto adquirida en la comunidad. Rev Chil Enf Respir 2005; 21: $111-6$

8.- RUIZ M, ARANCIBIA F, TORRES A. Epidemiología, factores de riesgo y pronóstico de la neumonía grave adquirida en la comunidad. En Torres A, Mensa J, Niederman MS. Infecciones respiratorias en UCI ed. Springer-Verlag Ibérica; 1999: 1-12.

9.- MINISTERIO DE SALUD. Guía clínica de neumonía adquirida en la comunidad en adultos de 65 años y más de manejo ambulatorio. Santiago, Ministerio de Salud, 2011.

10.- BANTAR C, CURCIO D, JASOVICH A, BAGNULO
H, ARANGO Á, BAVESTRELLO L, et al. Neumonía aguda adquirida en la comunidad en adultos: Actualización de los lineamientos para el tratamiento antimicrobiano inicial basado en la evidencia local del Grupo de Trabajo de Sudamérica (Consensur II). Rev Chil Infectol 2010; 27 (supl. 1): 9-38.

11.- INSTITUTO NACIONAL DE ESTADÍSTICA. Censo de Población y Vivienda, Chile, 2002. http://www.ine. cl/canales/chile_estadistico/censos_poblacion_vivienda/ censo_pobl_vivi.php (consultado el 21 de octubre de 2011).

12.- DAHMASH N, CHOWDHURY M. Re-evaluation of pneumonia requiring admission to an intensive care unit: a prospective study. Thorax 1994; 49: 71-6.

13.- SALDÍAS F, PAVIÉ J. Evaluación de la gravedad en la neumonía del adulto adquirida en la comunidad. Rev Chil Enf Respir 2005; 21:103-10.

14.- SALDÍAS F, MARDONEZ J, MARCHESSE M, VIVIANI P, FARÍAS G, DÍAZ A. Neumonía adquirida en la comunidad en el adulto hospitalizado. Cuadro clínico y factores pronósticos. Rev Med Chile 2002; 130: 1373-82.

15.- RIQUELME R, RIQUELME M, RIOSECO M, GÓMEZ V, CÁRDENAS G, TORRES C. Neumonía adquirida en la comunidad en el anciano hospitalizado. Aspectos clínicos y nutricionales. Rev Med Chile 2008; 136: 587-93.

16.- ESPAÑA P, CAPELASTEGUI A, QUINTANA J. Prospective comparison of severity scores for predicting clinically relevant outcomes for patients hospitalized with community-acquired pneumonia. Chest 2009; 135: 1572-9.
Correspondencia a:

Dr. Eduardo Cea V.

Gregorio Marañón 1416

Viña del Mar, V Región.

E-mail: eduardoceav@hotmail.com 